\title{
Téoros
}

Revue de recherche en tourisme

\section{Le rôle des institutions financières internationales : le cas du groupe de la Banque Mondiale}

\section{Charles-Étienne Bélanger}

Volume 13, numéro 2, été 1994

Regards sur le tourisme dans les pays en développement

URI : https://id.erudit.org/iderudit/1077758ar

DOI : https://doi.org/10.7202/1077758ar

Aller au sommaire du numéro

Éditeur(s)

Université du Québec à Montréal

ISSN

0712-8657 (imprimé)

1923-2705 (numérique)

Découvrir la revue

Citer cet article

Bélanger, C.-É. (1994). Le rôle des institutions financières internationales : le cas du groupe de la Banque Mondiale. Téoros, 13(2), 16-20.

https://doi.org/10.7202/1077758ar d'utilisation que vous pouvez consulter en ligne. 


\section{Le rôle des institutions financières internationales: le cas du groupe de la Banque Mondiale \\ Charles-Étienne Bélanger"}

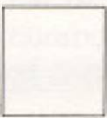

Le rôle des institutions financières internationales dans les pays en développement n'est jamais passé inaperçu. Pour le meilleur ou pour lepire, elles interviennent dans un grand nombre de pays et leur influencen'est pasnégligeable. Alors qu'on célèbre cette année le $50^{\mathrm{E}}$ anniversaire des accords de Bretton-Woods, plusieurs en profitent pour dresser un bilan des activités du Fonds Monétaire International (FMI) et de la Banque Mondiale qui virent le jour grâce à ces accords. Décriées par les uns, applaudies par les autres, ces institutions font plus que jamais l'objet de controverses ${ }^{(1)}$. Néanmoins, on ne peut passer sous silence leur apport dans un certain nombre de secteurs. Bien que marginal par rapport à d'autres domaines, le tourisme a a ussi été un secteur dont on s'est préoccupé à la Banque Mondiale. En voici une brève rétrospectiveoù nous verrons successivement dans quel contexte et pourquoi la Banque s'est intéressée au tourisme, puis nous retracerons les grandes étapes ayant caractérisé son intervention.

\section{L'émergence du tourisme dans le contexte du développement}

C'est au début des années 1960 qu'on voit surgir l'idée que le tourisme international peut aussi profiter aux pays en développement. Deux séries de facteurs expliquent en partie l'avènement de cette nouvelle conception. Il $y$ a d'une part les succès qu'ont connu plusieurs pays d'Europe occidentale en matière de tourisme international. Au lendemain du deuxième conflit mondial, bon nombre de ces pays étaient à la recherche de nouvelles sources de devises indispensables à la reconstruction de leurs économies. $\mathrm{La}$ conjoncture leur est favorable. En effet, ol'assouplissement [à partir de 1950] des réglementations appliquées à la fin de la Deuxième Guerre mondiale pour limiter la circulation des devises et les déplacements à l'étrangers('), la hausse des revenus dans les pays industrialisés et l'adoption de mesures sociales reconnaissant le droit auxvacances, favorisentl'essor dutourismeinternational. Cette réussite attire l'attention des institutions internationales telles les Nations Unies, I'UNESCO, I'OCDE, la Banque Mondiale etl'UIOOT ${ }^{(5)}$. D'autre part, il ya le fait que plusieurs pays en développement, nouvellement indépendants, deviennent membres de ces organismes internationaux et sont dès lors plus en mesure de pouvoir défendre leurs intérêts.

C'est dans ce contexte que les organisations internationales vont encourager le développement du tourisme comme une solution partielle aux problèmes des économies des pays en développement, notamment au chapitre de leur balance des paiements. La thèsevoulant quelespaysen développementpuissent profiter des devises qu'engendre l'arrivée de touristes pour équilibrer leur balance des paiements est officiellement appuyée par les Nations Unies en 1963 lorsque l'organisation affirme que ale tourisme peut apporter et apporte effectivement une contribution vitale à la croissance économique des PVD ${ }^{(4)}$. Cette thèse est également entérinée par la Banque Mondiale qui encourage l'élaboration et l'exécution de programmes de développement touristique.

\section{L'intérêt de la Banque Mondiale pour le tourisme}

L'intérêt de la Banque Mondiale pour le tourisme est significatif d'une double évolution: celle de la Banque dans sa recherche d'alternatives nouvelles en matière de développement économique et celle du phénomène touristique. La première se traduit par une prédisposition à orienter la politique économique des États vers le développement du tourisme, là où les conditions le justifient. La deuxième, appelle à profiter des opportunités qu'offre une industric en plein essor. Pour expliquer cet intérêt, des motifs d'ordre théorique et pratique doivent être considérés.
Monsieur Charles-Etienne Belanger est diplo Université du Québec à Montréal. II est actuélement directeur du Bits-Amériques. 


\section{La stratégie de la Banque}

Le tourisme en tant que facteur de développement apparait au début des années 1960. Ce n'est toutefois qu'en 1969 que la Banque Mondiale s'engage véritablement dans le développement de ce secteur en ouvrant un nouveau département chargé des projets touristiques. Cette décision repose sur la reconnaissance d'une croissance rapide du tourisme international et sur l'importance, pour un grand nombre de pays membres de la Banque, d'un secteur pourvoyeur de devises. Elle s'inscrit aussi dans le cadre des objectifs fixés par $\mathrm{McNamara}^{(5)}$ dans son programme quinquennal 1969-1973: doubler levolumedes engagements dans le monde, tripler le montant total des prêts à l'Afrique, réorienter les efforts tant sur le plan géographique que sectoriel.

La réalisation de ces objectifs n'efface toutefoispas les problèmes que connaissent les pays en développement. Malgré certaines améliorations (augmentation des investissements et des taux de croissance du PNB), on se rend vite compte de la persistance du chômage et du sous-emploi, du plus grand nombre de gens dans la pauvreté absolue et de l'inégalité dans la répartition des revenus. Ainsi voit-on apparaître au début des années 1970 un nouveau discours qui se dégage du seul critère - celui de la croissance du PNB ayant prévalu dans les activités de la Ban= que depuis de nombreuses années. S'inspirant des travaux de divers économistes ${ }^{(6)}$, ce discours est axé sur les problèmes de pauvreté et de répartition des revenus. Il marquera le second programme (19741978) de McNamara quis'en fera un vibrant défenseur comme en témoigne ses propos:

\section{Unepart importante du programme à mettre en oeuvre doits'attaquer à la pauvreté absolue, qui prend des proportions intolérables dans la plupart des pays du tiers monde... $O r$, le $P N B$ ne permet à lui seul de mesurer les résultats obtenus par rapport à des oljectifs de dévelop- pement multiples... [ill est un indice de lavaleur totale des biens et services prodaits par une économie; il n'a janais été conģu pour mesurer leur répartition.?}

En acoordant plus d'attention aux aspects sociaux de la croissance économique, la Banque va diversifier ses activités. Le

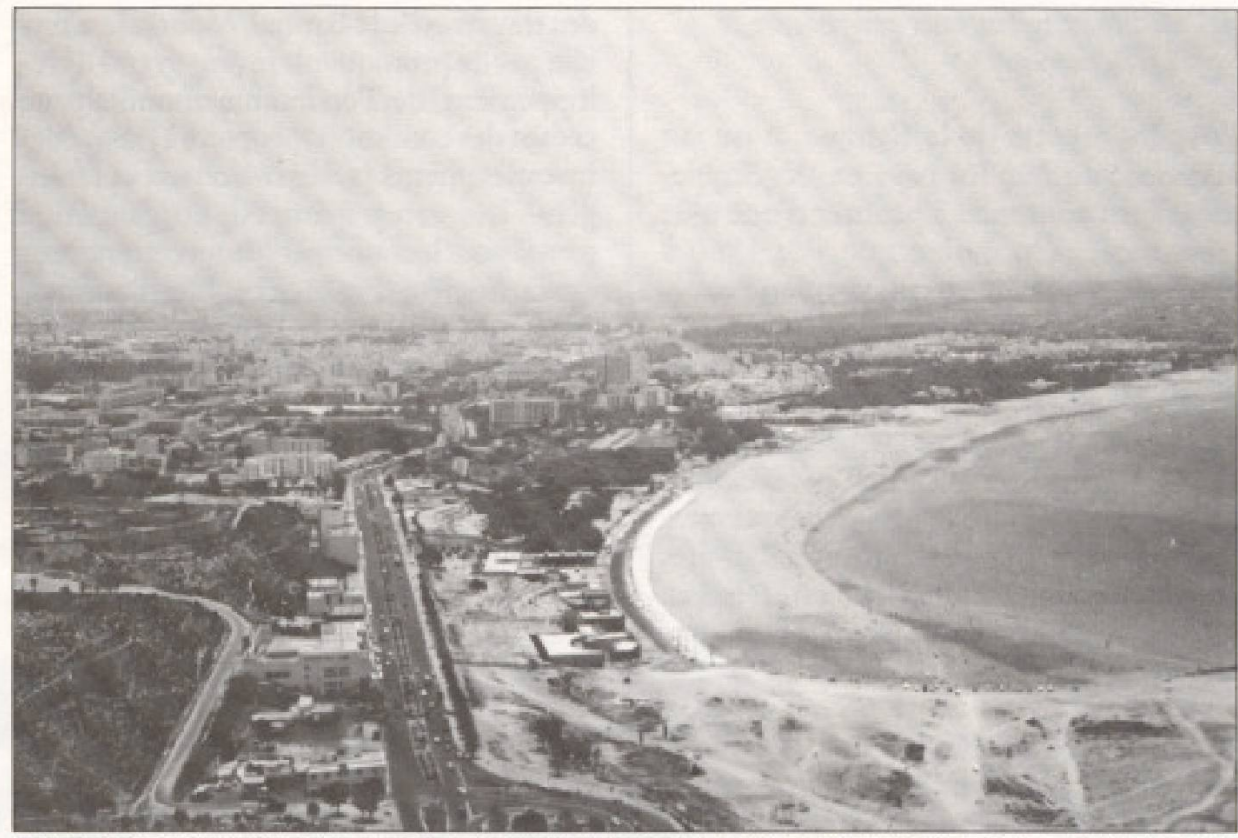

Vue de la baie d'Agadir au Maroc. Au cours des années 1970 , la Banque Mondiale y a financé dimportants travaux d'infrastructure pour développer le tourisme.

pourcentage des ressources financières consacré aux secteurs traditionnels (énergie, transport, télécommunications etautres projets d'infrastructure) sera réduit au profit de nouveaux secteurs: agriculture et développement rural, éducation, santé, urbanisation... et tourisme.

Les nouvelles priorités de la Banque Mondiale se reflètent dans la formulation des objectifs en matière de tourisme et dans les types de projets financés. L'apport en devises et la création d'emplois demeurent des objectifs essentiels, mais devant servir à la mise en valeur des régions moins développées des pays en développement et à l'atténuation des écarts entre les revenus et l'emploi d'une région à l'autre. La Banque se dit de plus préoccupée par les effets socio-culturels et environnementaux du tourisme dans les pays en développement et par certains aspects de la distribution des avantages que procure le tourisme :

Elle veut veiller à ce que les propriétaires privés ne soient pas les seuls à bénéficier de *pactoless lorsque la mise en valeur d'une nouvelle station balnéaire peut faire monter brusquement la valeur des terrains dans la région, (s)

Quant aux nouveaux types de projets, ils ne se limitent plus a la simple construction d'bôtels, mais portent aussi sur l'aménagement intégré de centres touristiques. Cette approcbe dite *intégrées se traduit par une aide de la Banque à des projets qui englobent plusieurs secteurs d'activites. On veut que ces types de projets puissent *contribuer a repartir plus largement les avantages du développenent, surtout parmiles couches patuvres de la sociétén(?).

Au-delà de tous ces aspects, la stratégie de la BanqueMondiale repose sur un élément qu'il convient de mettre en relief, car en bout de ligne, il est déterminant. Il s'agit de la croissance phénoménale de l'industrie touristique à l'échelle mondiale. Une croissance qui présente des opportunités rejoignant directement les intérêts de la Banque.

\section{Une industrie en plein essor}

Dans uneétude sectorielle publiééen 1972 , la Banque Mondiale rend compte des tendances du tourisme international. Les chiffres sont impressionnants:

De 1950 à 1970, les arrivées de visiteur étrangers (vacanciers, bommes d'affaires et autres) dans l'ensemble des pays sont pasoées de 25 à 168 millions, ce qui représente un taux de croissance annuel moyen de $10 \%$ et, au cours de la même période, les recettes au titre du tourisme international ont augmenté de 2,1 milliards de dollan à 17,4 milliards 


\section{de dollars, soit un taux de croissance de $11 \%$ par an, ${ }^{(10)}$}

Pour les experts de la Banque, il ne fait aucun doute que les pays en développement vont continuer à profiter d'une telle croissance. On évalue que *de 1960 à 1968 , alors que les exportations en provenance des pays en voie de développement (a l'exception des exportations pétrolières) augmentaient de $7,6 \%$ par an, les recettes provenant du tourisme augmentaient de $11 \%$ par an ${ }^{(11)}$. Et les perspectives d'avenir semblent tout aussi prometteuses. Particulièrement pour les flux touristiques en direction des Caraibes et du bassin méditerranéen où l'on table sur une forte augmentation.

Pour les pays méditerranéens, la Banque Mondialesignalequedesétudes consacrées auxmarchéseuropéen etaméricain laissent entrevoir qu'au cours des dix prochaines années, il devrait $\mathrm{y}$ avoir 10 millions de touristes supplémentaires, soitledoublede cequi prévalaiten 1968 dans cette zone. La hausse du niveau des revenus dans les pays émetteurs, l'évolution des habitudes de vacances et le développement du transport aérien offrant des tarifs abordables sont autant de facteurs responsables de la croissance de la demande touristique et de ses prévisions optimistes.

En contrepartie, les prévisions de la Banque concernant le développement de l'offre dans les pays en développement disposant d'un potentiel touristique, reposenten partie sur les perspectives incertaines d'un grand nombrede produits primaires sur les marchés mondiaux (limitation des importations en provenance des pays en développernent vers les pays industrialisés); ce qui devrait amener plusieurs pays à miser de plus en plus sur les recettes du tourisme. $\mathrm{La}$ croissance mondiale de l'industrie touristique et ses perspectives prometteuses pour les pays en développement, amèneront la Banque Mondiale à s'engager davantage dans un secteur qui lui permet de mettre en oeuvre ses stratégies en matière de développement économique.

\section{L'intervention de la Banque dans le secteur touristique}

Comme on a pu le voir, le tourisme offre des opportunités intéressantes à ceux qui désirent investir dans ce secteur, qu'il s'agisse d'investissements issus des pays en développement ou de l'étranger. Or, une

des missions de la Banque Mondiale n'estelle pas de promouvoir le plus grand développement de l'économie mondiale en créant des conditions propices à l'essor des investissements privés nationaux et étrangers?' Les statuts mêmes de l'organisation sontlà pour leconfirmer ${ }^{(1)}$ tout commeceux de la Société Financière Internationale (SFI), une filiale de la Banque qui investit dans le secteur touristique depuis 1967.

Son rôle, dès lors, consiste à établir une synergie entre les pays en développement désireux de développer le secteur touristique et les investisseurs privés qui désirenty investir des capitaux. Consciente des risquess liés à une main-misedes intérêts privés étrangerssur le développement touristique de certains pays en développement, la Banque définit avec précision son rôle:

\begin{abstract}
Encourager diverses formes d'entreprises conjointes qui permettraient aux pays en voie de developpement de garder une participation majoritaire dans l'équipement touristique et aux inveticeeurs étrangers d'engager des sommes suffisamment importantes pour garantir leur intérêt au succès de Pentreprise. (i)
\end{abstract}

C'est dans ces conditions que la Banque Mondiale et la SFI vont s'impliquer activement dans le secteur du tourisme. Une implication qui demeure toutefois négligeable lorsqu'on la compareaux autres secteurs d'activités où ces institutions sont présentes.

L'implication du Groupe de la Banque Mondiale ${ }^{(14)}$ dans le domaine du tourisme, qui remonte à la fin des années 1960 , va connaître plusjeurs changements. Si la SFI demeure toujours active dans le tourisme depuis qu'elle a approuvé son premier investissement pour le financementd'un hôtel en 1967, il n'en ira pas de même avec la Banque Mondiale qui va officiellement se retirer de ce secteur en juin 1979.

L'engagement de la Banque Mondiale en terme de soutien financier et d'assistance technique au secteur touristique est particulièrement fort au cours des années 1970 alors que la Banque opère un département spécial pour le financement de projets touristiques. Crééen 1969, cedépartement détermine et prépare des projets. Concrètement, le but recherché est d'aider les pays membres wà préparer des plans d'ensemble pour l'organisation du tourisme, à exécuter des études de justification économique pour chaque projet et à former descadres et spécialistes qualifiés, qu'il s'agisse de l'administration des programmes ou des opérations touristiques ellesmêmešn 19 .

Jusqu'en 1971, l'action du *Groupe de la Banque Mondiales (Banque Mondiale et SFI) se fait *principalement sous forme d'investissements et de prêts pour la SFI, et de financementd'hôtels par l'intermédiaire de sociétés financières de développements ${ }^{(16)}$. Mais, vers le milieu de l'année 1971, la décision de financer directement des projets touristiques est prise. Dorénavant, l'objectif est de favoriser les projets intégrés et multisectoriels axés sur les problèmes de développement des ressources humaines (programmes nationaux en faveur de l'emploi et du développement des services publics essentiels).

Le soutien financier de la Banque Mondiale(BIRD et IDA) au secteur du tourisme aurait été évalué à 450 millions de dollars US (volume cumulé des prêts, dont $87 \%$ viennent de l'IDA) sur un coût total des projets estimé à plus d'un milliard de dollars et ce, pour la période allant de 1971 à 1979(17).

Si ces chiffres semblent importants, il faut toutefois ajouter que le tourisme occupe une place marginale dans les opérations du Groupe de la Banque Mondiale, au cours de cette période, comme le démontrent les données suivantes ${ }^{(11)}$;

- $0,42 \%$ du total cumulé des prêts de I'IDA en juin 1980;

- $0,61 \%$ des prêts de la BIRD;

- $4,1 \%$ des investissements et prêts réalisés par la SFI (soit 58 millions de dollars);

- $1,4 \%$ du total des engagements de la BIRD et de l'IDA en 1977, année où les crédits accordés atteignent un maximum.

Entretemps, la Banque entreprend une réflexion sur la façon de développer le tourisme et sur les impacts de ce développement sur les sociétés d'accueil. Une publication parue en $1972^{(19)}$ tente de tirer des leçons de l'expérience. Ses constats portent autant sur l'aménagement de stations touristiques que sur l'impact économique de tels projets (les avantages doivent être évalués en fonction des coû́ts). Cette 


\section{TABLEAU 1}

Prêts et crédits de la Banque Mondiale appuyant le développement du tourisme entre 1989 et 1993

Pays Secteur(s)

Nombre de prêts/crédits
Année(s)

d'approbation
Montant (million $\boldsymbol{S}$ US)

\section{PRÊTS}

\begin{tabular}{ll}
\hline Egypte & $\begin{array}{l}\text { Infrastructure } \\
\text { touristique et gestion } \\
\text { environnementale } \\
\text { Environnement et } \\
\text { transport } \\
\text { Seychelles }\end{array}$ \\
Yougoslavie & $\begin{array}{l}\text { Modernisation du } \\
\text { réseau routier } \\
\text { Financement de } \\
\text { projets industriels } \\
\text { et touristiques } \\
\text { Infrastructure à } \\
\text { Belize City }\end{array}$ \\
\hline
\end{tabular}

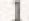

6

8

1

18
260,0

20

Total des prêts

746,5

\section{CRÉDITS}

\begin{tabular}{|c|c|c|c|c|}
\hline Kenya & $\begin{array}{l}\text { Zones protégées et } \\
\text { services de la faune }\end{array}$ & 1 & 1992 & 60,5 \\
\hline Maldives & $\begin{array}{l}\text { Modernisation de } \\
\text { l'aéroport }\end{array}$ & 1 & 1990 & 7,5 \\
\hline Tonga & $\begin{array}{l}\text { Banque de dévelop- } \\
\text { pement de Tonga }\end{array}$ & 1 & 1990 & 3,0 \\
\hline Total des crédits & & 3 & & 71,0 \\
\hline
\end{tabular}

Source: Banque Mondiale, 1994.

TABLEAU 2

Liste des investissements approuvés par la Société financière internationale entre 1967 et 1991

\begin{tabular}{ccccc}
\hline Période & $\begin{array}{c}\text { Nombre de } \\
\text { projets }\end{array}$ & $\begin{array}{c}\text { Coút total } \\
\text { des projets }\end{array}$ & $\begin{array}{c}\text { Investissement } \\
\text { brut* }\end{array}$ & $\begin{array}{c}\text { Investissement } \\
\text { net* }^{* *}\end{array}$ \\
\hline $1967-69$ & 4 & 19,1 & 7,9 & 6,7 \\
$1970-79$ & 18 & 147,6 & 37,6 & 29,5 \\
$1980-89$ & 33 & 645,2 & 178,2 & 155,8 \\
$1990-91$ & 23 & 708,3 & 188,4 & 132,0 \\
TOTAL & 78 & 1520,2 & 412,1 & 324,0 \\
& & & & (en millions \$ US) \\
\hline
\end{tabular}

* Ces montants incluent, en plus des investissements directs de la SFI, les prêts syndiqués par la SFI avec des banques commerciales internationales et d'autres participants.

* Ces montants représentent l'investissement direct de la SFI, sous forne de prêts ou de capital-actions.

Source: Société Financière Internationale. 
réflexion sera davantage approfondie lorsqu'en décembre 1976, la BanqueMondiale et l'UNESCO parrainent conjointement un cycle d'étude devant examiner l'impact social etculturel du tourismesur les PVD ${ }^{(20)}$.

En 1979, la Banque Mondiale prend la décisionde mettre un termeau financement direct de projets touristiques et ferme son département spécial. H.D. Davis et J.A. Simmons, anciens fonctionnaires de la Banque, expliquent cette décision de la façon suivante :

\section{The decision to phase out The department in 1979 was largely related to The increasing calls on limited Bank resources inother sectors, particularly for energy development, and to The recognition that in The preceeding decade The Bank badbeen able to belp a rather small minority of its developing country membership in The tourism field - only 18 out of about 120 countries. (2)}

Cette décision de mettre un frein aux prêts danslesecteur touristique correspond aussi à l'avènement des programmes dits d'ajustementstructurel qui entrenten scène à la faveur de la crise de l'endettement sévissant dans bon nombre de pays en développement au début des années 1980. Pour acoider aux ressources du Fonds Monétaire International (FMI), les pays doivent se soumettre aux conditions imposées par l'organisme. Cette politique dite de conditionnalité repose sur des critères de performance économique qui concernentautantledéficit publicquela fixation des prix, la restructuration ou la privatisation d'entreprises publiques, ou la gestion des devises et du taux de change. Cette nouvelle façon de faire va non seulement accroître le pouvoir d'intervention du FMI sur la politique économique des pays en développement, maiségalementinfluencer très fortement les prêts de la Banque Mondiale et le rééchelonnement dela dette auprès des Clubs de Paris et de Londres ${ }^{(22)}$.

\section{La situation actuelle}

Malgré les changements de priorités, la Banque Mondiale n'a jamais complètement cessé de financer des projets liés au tourisme. Comme l'illustre le tableau 1, plusieurs prêts ou crédits sont accordés à des projets ayant une incidence sur le secteur touristique. Les données fournies par la Banque Mondiale indiquent ainsi qu'au cours des cinq dernières années, soit entre 1989 et 1993, la Banque a approuvé des prêts totalisant près de 750 millions $\$$ US et a accordé des lignes de crédit pour une somme évaluéeà un peu plus de 70 millions SUS.

La Société Financière Internationale(SFI) demeure toutefois l'organe du groupe de la Banque Mondiale qui intervient le plus directement dans le secteur touristique. Le tourisme semble être un bon vecteur pour encourager la croissance du secteur privé, ce qui constitue la mission première de la SFI. En 1992, l'institution a d'ailleurs mis sur pied une unité spéciale affectée aux projets touristiques. Au cours des années, la SFI est intervenue dans plus d'une centaine de projets - surtout hôteliers - localisés dans une quarantaine de pays. Le tableau 2 présente la situation globale de 1967 à 1991 .

Comme on peut le constater, le nombre de projets financés par la SFI s'est accru rapidement au cours des années. Il en va de même du montant des investissements qui, même en tenant compte de l'inflation, a augmentéde façon trèsimportante, comme l'illustre les chiffres pour 1990-1991.

Bien que le tourisme n'ait jamais été reconnu comme un secteur prioritaire tel que l'agriculture, l'éducation ou la santé, il n'en demeure pas moins qu'il a fait l'objet d'une certaine attention de la part des institutions financières internationales. Aujourd'hui, conformément à la philosophie de la Banque Mondiale et du FMI, le financement de projets en tourisme vise d'abord et avant tout à encourager les investissements en provenance du secteur privé national. C'est là une façon relati= vement efficace de poursuivrel'intégration des pays en développement à l'ćoonomie mondiale.

\section{Notes}

(1) Voir entre autres l'article de Christian Ferrié, Est¿ possible de refomer la Banque Mandiale? Le Monde Diplomatique, juin 1994, pp. 18-19.

(2) Banque Mondiale. Tourisme. Étude sectorlelle, 1972 , p. 3.

(3) Union internationale des organismes officiels du tourisme quil deviendra en 1975 l"Organisation mondiale du tourisme [OMT).

14) United Nations, Conferencecon International Travel and tourism, Recommendations on International Travel and tourism, United Nations, 1953,p 17 (c'est nous qui traduisons).

(5) Larrivée de Robert S. McNamara a la tète de la Banque Mondiale en 1968 allait donner une nouvelle impulsion aux activités de l'institution. II en sera president jusqu'en 1981.

(6) Voir entre autres les travaux de Hollis B. Chenery et autres: Redistribution with Growth, Oxtord U.K., Oxford University Press, 1974. Le concept de sredistribution et croissances (Redistribution with Growth) deviendra en queloue sorte la marque de commerce de la Banque en 1974 . II suggẻe que les rewenus supplementaires de la croissance soient redirigés vers les pauvres plutöt que de revenir vers ceux qui sont déjà bien nantis. Dans la pratique, ce concept s'avéfer la toutefois difficilement applicable car il présuppose que tes Alites nationales fassent des concessions as plus défavorisés de la société, ce quiest bin d'stre évident.

(7) Discours prononoé devant le Conseil des Gouverneurs par fobert S. McNamara, président du Groupe de la Banque Mondiale, Nairobi, Kenya, 24 septembre 1973, pp, 13 et 32 .

(8) Banque Mondiale, Rapport annuel 1973, p. 26

(9) Ibid., p, 17

(10) Banque Mondiale, Tourisme (étude sectorielle) Wasthington, D.C., 1972, p. 4.

(11) lbid., p. 15.

(12) Dansl'article I des statuts de la BIRD oùl'on détinit les buts de l'organisation, on y lit que la Banque pour buts: *(ii) d'encourager l'investissement privé à l'étranger au moyen de garanties ou de participations aux emprunts et autres investisse. ments faits par des capital stes privés; en outre lorsque les captaux prives ne sontpas disponibles à des conditions rasisonnables, de fournir, à des conditions appropriées et pour des buts de production, des fonds prélevés sur son propre capital cuobtenus par son intermédiaire.... (iii) d'enooura. ger l'expansion équilibrée, al long terme, du commerce international et le maintien de l'équipement dans la balance des comptes, en encoura. geant l'investissement international pour le çéve. loppement des ressources productives des Etats. membres...4.

(13) Banque Mondiale. Tourisme (étude sectorielle). op. cit, p. 28.

(14) Lé Groupe de la Banque Mondiale englobe la Banque Mondiale lla Banque internationale pour la reconstruction et le déweloppement (BIRD) et son institution affiliee, I'Asssociation internationale de développement (IDA), la Société financière internationale (SFII, une filiale de la BIRD et l'Agence multilaterale de garantie des investissements (MIGA). La mission de chacune des institutions se présente ainsi: BIRD: crée en 1945 dans le but d'aider a reconstruite ot développer les econo. mies décimées par la guerre, la BIRD a comme principale mission ad'aider à relever les niveaux de vie dans les pays en développement en acheminant wers ces pays des ressources financières fournies par les pays develappés. (Banque Mon diale, Rapport annuel 1991); IDAc crééeen 1960, elle fournit une aide aux pays en développement (membres de la BIRD) plus pauves et ce, à des conditions pesant moins lourdement sur leur belance des paiements que les préts de la BIRD; SF| constituée en 1956, la SFI a comme premier roble d'encourager la croissance du secteur privé des pays en developpement en les aidant a mobilises les capitaux a l'intéfieur du pavs ou à l'étranger; MIGA: créte en 196e. lagence foumit un certain nombre de couvertures de risques politiques tels que révolutions, coups d'Etats, destructions ou fermetures causes par une guerre civile ou une insurrection, expropriation parl'Etat pour des motifs politiques, etc

(15) Banque Mondiale, Rapport annuel, 1970

(16) Banque Mondiale, Tour isme (átude sectorielle). op, cit. p, 18

(17) Voir.Jean-Simon Tabournel, we tourisme, pour ou contre le tiers monde? $\mathrm{s}$, Crolssance des jeunes nations, no 259 , mars 1984 , p. 25.

(19) Ibid., p. 25

(19) II s'agit de l'étude sectorielle sur le tourisme réalisêe par la Banque Mondiale.

(20) Les rechercheset discussions de ce cvcle d'étude feront l'objet d'une publication conjointe de la Banque Mondiale et de I'UNESCO: Tourisme, passeport pour le développement?, sous la direction d'Emanuel de Kadt

(21) H. David Davis et James A. Simmons, World Bank Experience with tourism Projects, tourism Management, vol. 3, no 4, décembre 1982, p. 212 .

(22) Le Club de Paris regroupe les créanciers publics alors que le Club de Londres regroupe les benques commerciales privees.

N.B. : L'essentiel de cet article provient du mémoire de maitrise de l'auteur : L'Etat marocain et sa poli. tique touristique : le róle des déterminants externes et internes (1960-1990) 